\title{
Efficient 2D double-quantum solid-state NMR spectroscopy with large spectral widths
}

Received 00th January 20xx, Accepted 00th January 20xx

DOI: $10.1039 / x 0 x \times 00000 x$

\author{
Katharina Märker, Sabine Hediger and Gaël De Paëpe*
}

www.rsc.org/

2D double-quantum single-quantum correlation spectra with arbitrary spectral widths can be recorded with SR26 and related supercycled recoupling sequences when applying SupercycleTiming-Compensation (STiC) phase shifts. This concept widely extends the applicability of supercycled sequences, most importantly for obtaining long-range distance constraints for structure determination with solid-state NMR.

Solid-state nuclear magnetic resonance (ssNMR) is a powerful spectroscopic technique which can provide unique structural information at atomic resolution for a wide variety of samples, ranging from biological macromolecules to inorganic materials. Valuable structural information can be obtained from dipolar recoupling experiments, ${ }^{1,2}$ because they reintroduce a coupling which is inversely proportional to the cube of the distance between the spins involved. Hence, such experiments give direct insight into spatial proximities and even allow internuclear distance measurements.

For homonuclear recoupling experiments, double-quantum (DQ) recoupling schemes are very useful because signals from uncoupled spins can be suppressed by an appropriate phase cycle ("DQ filter").3,4 This is essential when such contributions dominate the spectrum and conceal the desired signals from coupled spin pairs, as it is for instance the case when correlating nuclei with low natural isotopic abundance (NA), such as ${ }^{13} \mathrm{C}$ $(1.1 \% \mathrm{NA})$ or ${ }^{29} \mathrm{Si}(4.7 \% \mathrm{NA}) .{ }^{5,6}$ The feasibility of such experiments, which often suffer from very low sensitivity, has greatly increased in recent years, owing to the substantial sensitivity enhancements achievable with magic-angle spinning dynamic nuclear polarization (MAS-DNP). ${ }^{7,8}$

Efficient excitation and reconversion of $\mathrm{DQ}$ coherences is fundamental for a successful implementation of $D Q$ recoupling experiments. High DQ-filtered efficiencies ( 73\%) can in theory

Univ. Grenoble Alpes, CEA, CNRS, INAC, MEM, F-38000 Grenoble, France. E-mail: gael.depaepe@cea.fr

+ Electronic Supplementary Information (ESI) available: Experimental details, theory, distances in the ampicillin spectrum. See DOI: 10.1039/x0xx00000x be achieved using so-called $\gamma$-encoded recoupling sequences, ${ }^{9}$ for example POST-C7, ${ }^{10}$ SPC-5, ${ }^{11}$ R14, ${ }^{12}$ and CMRR. ${ }^{13}$ However, experimental imperfections and interfering interactions like chemical shift anisotropy (CSA) often lead to dramatically reduced efficiencies in practice, in particular over long recoupling times, which impairs the detection of long-distance correlations. ${ }^{14}$ Compensation for such deleterious influences can be achieved by supercycling where the same sequence is repeated with cyclic radio-frequency ( $r f$ ) phase shifts and/or permutations. ${ }^{15,16}$ Even though this often leads to a loss of the $\gamma$-encoding property and associated reduced theoretical maximum efficiency of $\sim 52 \%$, the increased robustness of supercycled sequences means that they frequently outperform $\gamma$-encoded sequences experimentally. ${ }^{16}$

While supercycled pulse schemes are thus well suited for challenging recoupling experiments, a major drawback exists with regard to the acquisition of two-dimensional (2D) homonuclear DQ-SQ correlation spectra, one of the most important applications of $D Q$ recoupling. When employing non$\gamma$-encoded DQ recoupling sequences and hence most supercycled ones, the indirect evolution time $\left(t_{1}\right)$ has to be rotor-synchronized in order to record clean spectra. This means that the maximum spectral width in the indirect $\left(F_{1}\right)$ dimension is limited to the MAS frequency, which is often insufficient for nuclei with a broad chemical shift range. This limitation becomes even more severe at high magnetic fields, since the chemical shift dispersion in $\mathrm{Hz}$ is proportional to the magnetic field. For instance, a typical ${ }^{13} \mathrm{C}$ chemical shift range of $160 \mathrm{ppm}$ requires a $D Q$ chemical shift range of up to $320 \mathrm{ppm}$. This translates into a $F_{1}$ spectral width, and hence a required MAS frequency, of $\sim 32 \mathrm{kHz}$ at $9.4 \mathrm{~T}$, or $\sim 64 \mathrm{kHz}$ at $18.8 \mathrm{~T}$. Such high spinning frequencies are however incompatible with most $D Q$ excitation schemes and/or the available probe hardware. An insufficient $F_{1}$ spectral width results in signal folding in the DQ dimension whereas non-rotor-synchronized $t_{1}$ evolution gives rise to reconversion rotor-encoded DQ spinning sidebands, 17,18 in either way leading to difficulties in interpreting these more complex spectra. Note that this limitation can be circumvented 
for $y$-encoded sequences by applying appropriate phase shifts to the DQ reconversion pulses (vide infra), allowing the choice of a $t_{1}$ increment of arbitrary length. ${ }^{19-22}$

In this communication, we show that 2D DQ-SQ spectra with large $F_{1}$ spectral widths can be recorded with non- $\gamma$-encoded supercycled DQ recoupling sequences if they are derived from $\gamma$-encoded sequences. The method is based on SupercycleTiming-Compensation (STiC) phase shifts. It eliminates one of the biggest drawbacks of supercycling, enabling 2D correlations in samples with a wide chemical shift range without limitations regarding the size of the $D Q$ dimension. This is of particular relevance for the recoupling of nuclei with large CSA and/or small dipolar couplings, where well-compensated sequences are required. It also recently enabled us to complete the resonance assignment of a 2'-deoxyguanosine derivative (170 ppm ${ }^{13} \mathrm{C}$ chemical shift range) through the use of a ${ }^{13} \mathrm{C}-{ }^{13} \mathrm{C}$ correlation spectrum ${ }^{23}$ recorded with the SR26 recoupling sequence. ${ }^{24}$ In the following, we will describe the concept and derivation of the STiC phase shifts. It will be demonstrated on the SR26 pulse scheme, one of the most robust homonuclear DQ recoupling sequences to date. ${ }^{24,15}$

The SR26 sequence is a supercycled sequence constructed from the symmetry-based $\mathrm{R} 26_{4}^{11}$ pulse scheme. ${ }^{12,22,24}$ It consists of the R26 $6_{4}^{11}$ sequence $\left(S_{0}\right)$, a $\pi$-shifted cycle in which all $r f$ phases are shifted by $180^{\circ}\left(S_{\pi}\right)$, a phase-inverted cycle in which the signs of all rf phases are inverted $\left(S_{0}^{\prime}\right)$, and a $\pi$-shifted phaseinverted cycle which combines both operations $\left(S_{\pi}^{\prime}\right) \cdot{ }^{24}$ The full sequence $\left(S_{0} S_{0}^{\prime} S_{\pi}^{\prime} S_{\pi}\right.$, Fig. 1 b) spans 16 rotor periods $\left(\tau_{r}\right)$ and requires an $\mathrm{rf}$ nutation frequency of 6.5 times the MAS frequency. Its high robustness has for instance enabled the acquisition of ${ }^{29} \mathrm{Si}-{ }^{29} \mathrm{Si} \mathrm{DQ}$ build-up curves of zeolite and silicate samples at NA, which were sensitive to Si-Si distances of up to $8 \AA^{25}$ and played an important role in structure elucidation of these systems. ${ }^{26-28}$

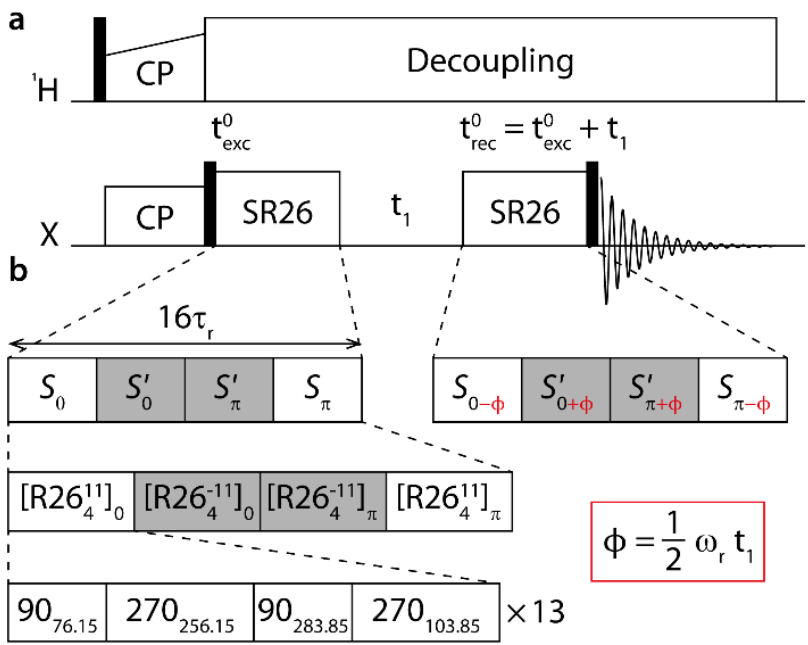

Fig. 1. (a) Pulse sequence for the acquisition of a $2 D D Q-S Q$ correlation spectrum with SR26 used as dipolar recoupling sequence, with $\mathrm{X}$ being, e.g., ${ }^{13} \mathrm{C},{ }^{15} \mathrm{~N}$ or ${ }^{29} \mathrm{Si}$. (b) Construction scheme of SR26. ${ }^{24}$ The sequence consists of four $S$ elements based on the $\mathrm{R} 26_{4}^{11}$ sequence. Overall rf phase shifts are indicated by subscripts, phase inversion by $S^{\prime}$ and gray boxes. The construction of one R-element is given in the bottom boxes, with flip angles and phases (subscript) in degrees. STiC phase shifts $\phi$ (highlighted in red) have to be applied to the DQ reconversion rf pulses in order to allow the use of a $t_{1}$ increment of arbitrary length.
A standard 2D DQ-SQ correlation experiment consists of a DQ excitation block, followed by $t_{1}$ evolution and a DQ reconversion block, usually of the same length as the $D Q$ excitation (Fig. 1a). When a rotor-synchronized DQ recoupling sequence is used, the position of the rotor should be the same at the starting point of $\mathrm{DQ}$ excitation $\left(t_{\text {exc }}^{0}\right)$ and of $\mathrm{DQ}$ reconversion $\left(t_{r e c}^{0}\right)$ to avoid modulation of the recoupled signal by sample rotation. ${ }^{17,18}$ This means that the $t_{1}$ evolution interval has to be an integer multiple of a rotor period, restricting the maximum $F_{1}$ spectral width to the spinning frequency. For $\gamma$-encoded sequences, this restriction can be circumvented by removing the dependence of the recoupled Hamiltonian on $t_{\text {rec }}^{0}$. This is accomplished by shifting the phases of all DQ reconversion $\mathrm{rf}$ pulses $\phi_{\text {rec }}$ according to the phase-time relationship ${ }^{21,22}$

$$
\phi_{r e c}=\phi_{e x c}+\frac{m}{\mu} \omega_{r}\left(t_{e x c}^{0}+t_{1}\right)
$$

where $m$ and $\mu$ are quantum numbers of the symmetry-allowed first-order terms, and $\omega_{r}$ is the angular spinning frequency. The applicability of this relationship requires that $\mu \neq 0$, and that $m / \mu$ is the same for all symmetry-allowed terms, which is only the case for $y$-encoded sequences. For detailed accounts of symmetry-based recoupling sequences and derivation of the relationships presented here, please refer to the literature ${ }^{22}$ and the ESI.

The selection rules for $\mathrm{R} N_{n}^{v}$ sequences can be applied to determine which terms are symmetry-allowed by the SR26 sequence. ${ }^{12}$ To first order, the $S_{0}$ and $S_{\pi}$ elements allow dipoledipole terms with quantum numbers $\{l, m, \lambda, \mu\}=$ $\{2, \pm 1,2, \mp 2\}$, whereas the related phase-inverted elements $S_{0}^{\prime}$ and $S_{\pi}^{\prime}$ allow $\{2, \pm 1,2, \pm 2\}$ terms. Since the ratio $m / \mu$ is not the same for all $S$ elements, the SR26 sequence is not $\gamma$-encoded. However, each separate $S$ element is still $\gamma$-encoded, having $m / \mu$ ratios of $-1 / 2\left(S_{0}, S_{\pi}\right)$ or $+1 / 2\left(S_{0}^{\prime}, S_{\pi}^{\prime}\right)$, respectively. As described above, this property can be used to remove the $t_{\text {rec }}^{0}{ }^{-}$ dependence of the average Hamiltonians created by the different R26 cycles. Assuming $t_{\text {exc }}^{0}=0$, the following overall phase shifts $\Delta \phi$ have to be applied to the DQ reconversion pulses of the respective $S$ elements, in addition to the phase shifts for DQ coherence selection:

$$
\begin{aligned}
& \Delta \phi\left(S_{0}\right)=\Delta \phi\left(S_{\pi}\right)=\phi_{r e c}\left(S_{0}\right)-\phi_{\text {exc }}\left(S_{0}\right)=-1 / 2 \omega_{r} t_{1} \\
& \Delta \phi\left(S_{0}^{\prime}\right)=\Delta \phi\left(S_{\pi}^{\prime}\right)=\phi_{r e c}\left(S_{0}^{\prime}\right)-\phi_{\text {exc }}\left(S_{0}^{\prime}\right)=+1 / 2 \omega_{r} t_{1}
\end{aligned}
$$

This makes all four separate average Hamiltonians independent of $t_{r e c}^{0}$, and hence also their sum, which is the effective Hamiltonian of the SR26 sequence. Consequently, arbitrary $t_{1}$ increments can be used in a DQ-SQ correlation experiment with SR26, and any desired $F_{1}$ spectral width can be achieved, when these Supercycle-Timing-Compensation (STiC) phase shifts are applied (also highlighted in Fig. 1b). The concept of these phase shifts can be transferred to similar supercycles of other sequences, as long as their separate cycles are $y$-encoded.

Fig. 2a demonstrates the successful application of STiC phase shifts in a ${ }^{13} \mathrm{C}-{ }^{13} \mathrm{C}$ DQ-SQ correlation experiment of a DNPenhanced frozen solution of $U-{ }^{13} \mathrm{C},{ }^{15} \mathrm{~N}$-labeled L-histidine. The spectrum was taken at a spinning frequency of $7.8 \mathrm{kHz}$, 
employing the SR26 pulse sequence as given in Fig. 1. A $t_{1}$ increment of $33.3 \mu \mathrm{s}$, which is much shorter than a rotor period $\left(\tau_{r}=128.2 \mu \mathrm{s}\right)$, was used to achieve an $F_{1}$ spectral width of $30 \mathrm{kHz}$. The spectrum is dominated by cross peaks between directly bonded carbons, located in $F_{1}$ at the sum of the chemical shifts of the respective correlated spins, and allows to establish carbon-carbon connectivities (green solid lines). In addition, peaks originating from relayed polarization transfer over two bonds can be observed (green diamonds), which is typical for dipolar recoupling experiments with longer mixing times in uniformly ${ }^{13} \mathrm{C}$-labeled samples. ${ }^{20}$ Direct polarization transfers over two bonds are only observed for correlations involving the isolated $\mathrm{C} \epsilon$ with either $C_{\gamma}$ or $\mathrm{C} \delta$ (green dotted lines).

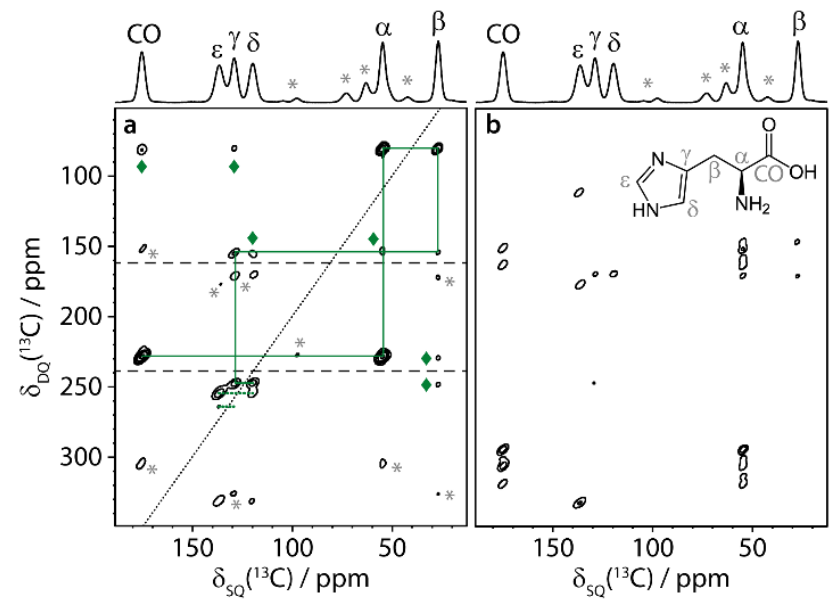

Fig. 2. DNP-enhanced ${ }^{13} \mathrm{C}-{ }^{13} \mathrm{C}$ DQ-SQ correlation spectra of a solution of $\mathrm{U}^{-13} \mathrm{C},{ }^{15} \mathrm{~N}-\mathrm{L}-$ histidine in $60 / 30 / 10(\mathrm{v} / \mathrm{v} / \mathrm{v}) \mathrm{D}_{8}$-glycerol/ $\mathrm{D}_{2} \mathrm{O} / \mathrm{H}_{2} \mathrm{O}$, containing $10 \mathrm{mM}$ AMUPol. ${ }^{29}$ The pulse sequence shown in Fig. 1 was used with (a) and without (b) STiC phase shifts. Spectra were acquired at $7.8 \mathrm{kHz}$ MAS frequency $\left(\tau_{r}=128.2 \mu \mathrm{s}\right)$, using a $t_{1}$ dwell time of $33.3 \mu \mathrm{s} \mathrm{(30} \mathrm{kHz} F_{1}$ spectral width) and a total recoupling time of $4.1 \mathrm{~ms}$ (see ESI for experimental details). Identical processing has been applied to both spectra, and the same contour levels are shown. Solid and dotted green lines in (a) illustrate direct one and two bond connections, respectively, and green diamonds indicate peaks originating from relayed transfers. Black horizontal dashed lines indicate the maximum $F_{1}$ spectral width feasible with rotor-synchronized $t_{1}$ evolution at the MAS frequency and magnetic field $(9.4 \mathrm{~T})$ used here. ${ }^{13} \mathrm{C}$-CPMAS spectra are displayed above for illustrational purposes. Asterisks denote spinning sidebands and glycerol signals.

For comparison, a spectrum taken with identical parameters, but without STiC phase shifts $(\Delta \phi=0)$, is shown in Fig. 2 b. Here, cross peaks are of lower intensity and do not appear at the expected $D Q$ frequency, but rather at more than one $D Q$ frequency for the same ${ }^{13} \mathrm{C}-{ }^{13} \mathrm{C}$ pair. The analysis of such a spectrum is practically impossible.

Due to its high robustness, the SR26 sequence is particularly well suited to the observation and measurement of long distances (>3 $\AA$ ), and thus the extraction of important structural information. Such polarization transfer over long distances is greatly facilitated in samples with nuclei at low NA or with sparse isotopic labeling since the relevant spin pairs occur mostly isolated and dipolar truncation is greatly reduced. ${ }^{25,30-32}$ This case is exemplified in Fig. 3 which shows a DNP-enhanced ${ }^{13} \mathrm{C}^{-13} \mathrm{C}$ DQ-SQ spectrum of the antibiotic ampicillin at its NA. The spectrum was recorded at a MAS frequency of $6 \mathrm{kHz}$, employing the SR26 sequence as shown in Fig. 1 . The large $F_{1}$ spectral width of $26.2 \mathrm{kHz}$ was achieved by using a non-rotorsynchronized $t_{1}$ increment of $38.2 \mu \mathrm{s}\left(\tau_{r}=166.7 \mu \mathrm{s}\right)$. Three repetitions of the full SR26 cycle were used for DQ excitation and reconversion each, resulting in a total recoupling time of $16 \mathrm{~ms}$. All expected correlations corresponding to one ( $1.5 \AA ̊)$ and two $(\sim 2.5 \AA)$ bond distances are present in the spectrum (yellow and orange dashed lines, respectively), but more importantly, numerous correlations over longer distances can be observed (red and blue solid lines). Comparison to the crystal structure ${ }^{33}$ shows that they range from $3.1 \AA$ to $\sim 4.3 \AA$, as also summarized in the ESI. Notably, not only intramolecular contacts are detected (red), but also several intermolecular ones (blue), which provide information on the crystal packing of the substance. Many of these correlations involve carbonyl and/or aromatic carbons which is remarkable due to their large CSAs and demonstrates the good performance of the SR26 sequence.

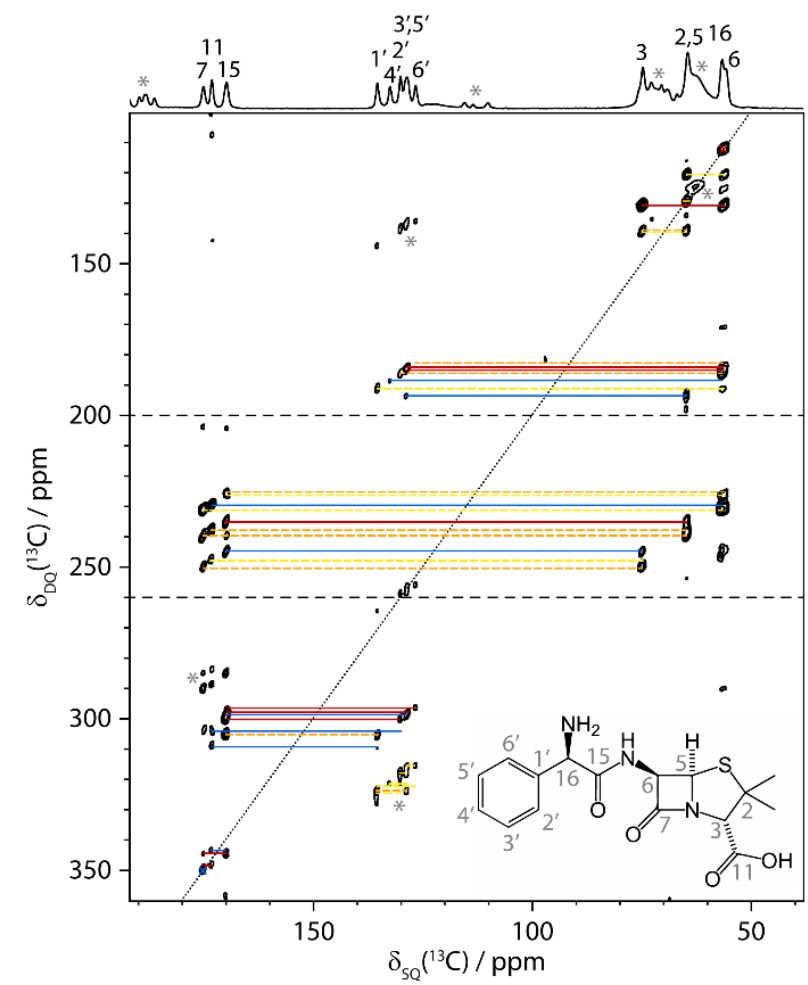

Fig. 3. DNP-enhanced ${ }^{13} C_{-13}{ }^{13}$ DQ-SQ correlation spectrum of NA ampicillin anhydrate, employing the SR26 sequence for dipolar recoupling as displayed in Fig. 1. The spectrum was recorded at $6 \mathrm{kHz}$ MAS frequency with a total recoupling time of $16 \mathrm{~ms}$ (see ESI for experimental details). Yellow and orange dashed lines denote correlations corresponding to one and two bonds, respectively. Solid lines indicate longer intra- (red) and intermolecular (blue) correlations (3.1 - 4.3 $\AA$, see also ESI)..$^{33}$ Only the shortest distance contribution is marked for each pair of cross peaks. Black dashed lines indicate the maximum $F_{1}$ spectral width feasible with rotor-synchronized $t_{1}$ evolution at the MAS frequency and magnetic field (9.4 T) used here. $\mathrm{A}^{13} \mathrm{C}$-CPMAS spectrum is displayed above for illustrational purposes, with resonance assignment taken from ref. ${ }^{34}$. Asterisks denote spinning sidebands and signals from glycerol. The most intense aromaticaromatic correlation peaks occur at the position of the $\left(\omega_{D Q}+\omega_{r}\right) / 2 \pi$ spinning sideband in the indirect dimension, caused by insufficient averaging of their large CSA at $6 \mathrm{kHz}$ MAS frequency. Correlations involving the methyl groups cannot be detected, which is often the case at measurement temperatures of $\sim 105 \mathrm{~K} .{ }^{35}$ 
In both examples presented here, the maximum $F_{1}$ spectral width that would have been attainable with rotor-synchronized $t_{1}$ evolution is marked by black horizontal dashed lines (Fig. 2a and 3). It is obvious that a spectrum with such a small spectral width and hence folding of many cross peaks would be extremely difficult to interpret, even for the simple case of histidine. Furthermore, the spectra shown here were recorded at a relatively low magnetic field of $9.4 \mathrm{~T}$. The available $F_{1}$ spectral width with rotor-synchronized $t_{1}$ evolution would be even smaller at higher magnetic fields.

In summary, we have presented a method for recording 2D DQSQ correlation spectra with arbitrary $F_{1}$ spectral widths when employing the supercycled symmetry sequence SR26. It is based on applying STiC phase shifts to the DQ reconversion pulses in order to compensate for non-rotor-synchronized $t_{1}$ evolution, extending the concept known for $\gamma$-encoded sequences. Consequently, all correlation peaks of samples with a large chemical shift range can be obtained without signal folding in the indirect dimension, but also experimental time can be saved when an $F_{1}$ spectral width smaller than the MAS frequency is sufficient. The recoupling performance of SR26 remains unchanged, meaning that all benefits of this well-compensated sequence can be exploited, as for example for the recoupling of nuclei with large CSA, and for the detection of long distance correlations for structural studies. This will be particularly useful for MAS-DNP applications involving the acquisition of 2D correlation spectra of dilute spins, as demonstrated here by a long-range ${ }^{13} \mathrm{C}-{ }^{13} \mathrm{C}$ correlation spectrum of NA ampicillin. The general principle of applying different $t_{1}$-dependent phase shifts to each element of a supercycle is applicable to any conventional supercycle of a $\gamma$-encoded sequence, solving one of the major problems associated with supercycle construction. This work was supported by the French National Research Agency (ANR-12-BS08-0016-01, ANR-11-LABX-0003-01 and RTB) and the European Research Council (ERC-CoG-2015, No. 682895). We are grateful to Daniel Lee and Nghia Tuan Duong for initial discussions about SR26, and to Sachin R. Chaudhari for preparation of the ampicillin sample. There are no conflicts of interest to declare.

\section{References}

1 G. De Paëpe, Annu. Rev. Phys. Chem., 2012, 63, 661-684.

2 N. C. Nielsen, L. A. Strass $\varnothing$ and A. B. Nielsen, Top. Curr. Chem., 2012, 306, 1-45.

3 A. Wokaun and R. R. Ernst, Chem. Phys. Lett., 1977, 52, 407-412.

4 R. Tycko and G. Dabbagh, J. Am. Chem. Soc., 1991, 113, 94449448.

5 A. Bax, R. Freeman and S. P. Kempsell, J. Am. Chem. Soc., 1980, 102, 4849-4851.

6 A. Bax, R. Freeman and T. A. Frenkiel, J. Am. Chem. Soc., 1981, 103, 2102-2104.

7 D. A. Hall, D. C. Maus, G. J. Gerfen, S. J. Inati, L. R. Becerra, F. W. Dahlquist and R. G. Griffin, Science, 1997, 276, 930-932.

8 D. Lee, S. Hediger and G. De Paëpe, Solid State Nucl. Magn. Reson., 2015, 66, 6-20.

9 N. C. Nielsen, H. Bildsøe, H. J. Jakobsen and M. H. Levitt, J. Chem.
Phys., 1994, 101, 1805-1812.

10 M. Hohwy, H. J. Jakobsen, M. Edén, M. H. Levitt and N. C. Nielsen, J. Chem. Phys., 1998, 108, 2686-2694.

11 M. Hohwy, C. M. Rienstra, C. P. Jaroniec and R. G. Griffin, J. Chem. Phys., 1999, 110, 7983-7992.

12 M. Carravetta, M. Edén, X. Zhao, A. Brinkmann and M. H. Levitt, Chem. Phys. Lett., 2000, 321, 205-215.

13 G. De Paëpe, J. R. Lewandowski and R. G. Griffin, J. Chem. Phys., 2008, 128, 124503-124526.

14 T. Karlsson, J. M. Popham, J. R. Long, N. Oyler and G. P. Drobny, J. Am. Chem. Soc., 2003, 125, 7394-7407.

15 P. E. Kristiansen, M. Carravetta, J. D. van Beek, W. C. Lai and M. H. Levitt, J. Chem. Phys., 2006, 124, 234510-234519.

16 M. Edén, in eMagRes, John Wiley \& Sons, Ltd, Chichester, UK, 2013, pp. 351-364.

17 H. Geen, J. J. Titman, J. Gottwald and H. W. Spiess, J. Magn. Reson. Ser. A, 1995, 114, 264-267.

18 U. Friedrich, I. Schnell, S. P. Brown, A. Lupulescu, D. E. Demco and H. W. Spiess, Mol. Phys., 1998, 95, 1209-1227.

19 T. Karlsson, A. Brinkmann, P. J. E. Verdegem, J. Lugtenburg and M. H. Levitt, Solid State Nucl. Magn. Reson., 1999, 14, 43-58.

20 A. Brinkmann, M. Edén and M. H. Levitt, J. Chem. Phys., 2000, 112, 8539-8554.

21 A. Brinkmann, PhD thesis, Stockholm University, 2001.

22 M. H. Levitt, Encycl. Nucl. Magn. Reson., 2002, 9, 165-196.

23 K. Märker, M. Pingret, J.-M. Mouesca, D. Gasparutto, S. Hediger and G. De Paëpe, J. Am. Chem. Soc., 2015, 137, 13796-13799.

24 P. E. Kristiansen, M. Carravetta, W. C. Lai and M. H. Levitt, Chem. Phys. Lett., 2004, 390, 1-7.

25 D. H. Brouwer, P. E. Kristiansen, C. A. Fyfe and M. H. Levitt, J. Am. Chem. Soc., 2005, 127, 542-543.

26 D. H. Brouwer, R. J. Darton, R. E. Morris and M. H. Levitt, J. Am. Chem. Soc., 2005, 127, 10365-10370.

27 D. H. Brouwer, S. Cadars, J. Eckert, Z. Liu, O. Terasaki and B. F. Chmelka, J. Am. Chem. Soc., 2013, 135, 5641-5655.

28 S. Cadars, M. Allix, D. H. Brouwer, R. Shayib, M. Suchomel, M. N. Garaga, A. Rakhmatullin, A. W. Burton, S. I. Zones, D. Massiot and B. F. Chmelka, Chem. Mater., 2014, 26, 6994-7008.

29 C. Sauvée, M. Rosay, G. Casano, F. Aussenac, R. T. Weber, O. Ouari and P. Tordo, Angew. Chem., Int. Ed., 2013, 52, 1085810861.

30 H. Takahashi, D. Lee, L. Dubois, M. Bardet, S. Hediger and G. De Paëpe, Angew. Chem., Int. Ed., 2012, 51, 11766-11769.

31 G. Mollica, M. Dekhil, F. Ziarelli, P. Thureau and S. Viel, Angew. Chem., Int. Ed., 2015, 54, 6028-6031.

32 K. Märker, S. Paul, C. Fernández-de-Alba, D. Lee, J.-M. Mouesca, S. Hediger and G. De Paëpe, Chem. Sci., 2017, 8, 974-987.

33 M. O. Boles and R. J. Girven, Acta Crystallogr. Sect. B, 1976, 32, 2279-2284.

34 O. N. Antzutkin, Y. K. Lee and M. H. Levitt, J. Magn. Reson., 1998, 135, 144-155.

35 V. S. Bajaj, P. C. A. van der Wel and R. G. Griffin, J. Am. Chem. Soc., 2009, 131, 118-128. 